\title{
Opportunity for catch-up HPV vaccination in young women after first delivery
}

\author{
Cristina Helena Rama, ${ }^{1,2}$ Luisa L Villa, ${ }^{3}$ Sonia Pagliusi, ${ }^{4}$ Maria A Andreoli, ${ }^{3}$ \\ Maria C Costa, ${ }^{3}$ Patricia Thomann, ${ }^{5}$ Venancio A F Alves, ${ }^{2}$ Adhemar Longatto-Filho, ${ }^{6,7}$ \\ Jose Eluf-Neto ${ }^{2}$
}

${ }^{1}$ Hospital Maternidade Leonor Mendes de Barros, São Paulo, 1 Brazil

${ }^{2}$ University of São Paulo School of Medicine, São Paulo, Brazil

${ }^{3}$ Ludwig Institute for Cancer Research, São Paulo, Brazil ${ }^{4}$ Ludwig Institute for Cancer Research, Lausanne,

Switzerland

${ }^{5}$ Oiagen do Brasil, São Paulo, Brazil

${ }^{6}$ Life and Health Sciences

Research Institute (ICVS),

School of Health Sciences,

University of Minho, Braga,

Portugal

${ }^{7}$ Pathology Division, Adolfo Lutz Institute

\section{Correspondence to}

Cristina Helena Rama, Hospital Maternidade Leonor Mendes de Barros, Av Celso Garcia, 2477. 03015-000 Belenzinho São Paulo-SP, Brazil;

crisrama@usp.br

Accepted 6 July 2009

\section{ABSTRACT}

Background Early age at first delivery has been identified as a risk factor for high-risk HPV-type infection and cervical cancer development.

Methods A cross-sectional study was carried out in a large public maternity hospital in São Paulo, Brazil. During June 2006 to February 2007, 301 women aged 15-24 years who gave birth to their first child were recruited between 43 and 60 days after delivery. Detection of HPV DNA in cervical specimens was performed using a standardised PCR protocol with PGMY09/11 primers. The association of selected factors with HPV infection was assessed by using a Generalised Linear Model.

Results HPV DNA was detected in $58.5 \%(95 \% \mathrm{Cl}$ $52.7 \%$ to $64.0 \%$ ) of the enrolled young women. The most common types of HPV found were: HPV16, HPV51, HPV52, HPV58 and HPV71. The overall prevalence of HPV types targeted by the HPV prophylactic vaccines was: HPV 16-12.0\%, HPV 18- 2.3\% and HPV 6 and 11 4.3\%. In the multivariate analysis, only age (inversely, $p$ for trend $=0.02$ ) and smoking habits were independently associated with HPV infection.

Conclusions The findings show that these young primiparous women had high cervical HPV prevalence, suggesting that this is a high-risk group for cervical cancer development. Nevertheless, $17.3 \%$ were positive for any of the four HPV types included in HPV vaccines (HPV6, 11, 16 or 18), with $13.3 \%$ positive for HPV 16 or 18 and only $1.0 \%$ having both vaccine related-oncogenic HPV types. Thus, young primiparous women could benefit from catch-up HPV vaccination programmes.

\section{INTRODUCTION}

Genital infection by oncogenic human papillomavirus (HPV) is a necessary factor in the development of cancer of the cervix. ${ }^{1}$ Worldwide, approximately $80 \%$ of around 500000 of registered cervical cancer cases affect women in developing countries, and some 20000 of them occur each year in Brazil. ${ }^{2}$ Although the HPV family of viruses includes more than 100 different viral genotypes, ${ }^{3}$ types 16 and 18 were identified in about $70 \%$ of cervical cancer cases. ${ }^{4}$

Vaccines to prevent infections by high-risk viral genotypes 16 and 18 have been developed and have an excellent safety, immunogenicity and efficacy profile for the prevention of HPV infections, cytologic lesions and high-grade cervical lesions in women. 56

Ideally, HPV vaccination should take place before potential exposure to HPV through sexual contact, ${ }^{7}$ because HPV L1 virus-like particle-based vaccina- tion has no therapeutic efficacy. ${ }^{8}$ In some countries school vaccination programmes have been established as a strategy to vaccinate girls against HPVassociated diseases including cervical cancer. However, girls of lower socioeconomic status are more likely to drop out of school early. ${ }^{9}$

In addition, vaccine coverage in adolescents can be low because they generally seek recommended preventive health services less frequently than other age groups. ${ }^{7}$ Furthermore, early age at first delivery has been identified as a risk factor for cervical cancer development. ${ }^{10}$

Therefore, opportunities for catch-up vaccination are valuable to improve coverage among the at-risk young female population. One such potential opportunity is offered by healthcare provided at first delivery; this approach would reduce the costs of reaching the 'hard-to-reach' adolescent groups by using infrastructures and procedures already in place in health services for offering novel vaccines. 2

However, the success of catch-up vaccination of young women after first delivery depends on the baseline prevalence of high-risk genotypes 16 and 18 in this target group.

So far, HPV infection rates have been shown to vary from $10.1 \%$ to $37.2 \%$ in pregnant women ${ }^{11-13}$ in previous reports that used different HPV detection techniques, distinct periods of pregnancy when samples were collected, and included participants in different age ranges, but did not focus on primiparous women. In particular, few studies in the postnatal period have reported vaccine-related HPV genotype prevalence in healthy young women.

Therefore, the aim of this study was to determine cervical type-specific HPV DNA prevalence and risk factors associated with HPV infection after the delivery of the first child among low-income young women in a public maternity hospital in the city of São Paulo, Brazil.

\section{MATERIAL AND METHODS Study population}

This cross-sectional study was carried out at Hospital Maternidade Leonor Mendes de Barros (HMLMB), one of the largest public maternity hospitals in São Paulo, Brazil. Primiparous women aged between 15 and 24 years who had been living in the metropolitan area of São Paulo for at least 6 months, and gave birth at this hospital after more than 32 weeks of gestation, were eligible for the study. They were enrolled at their postnatal visit, from June 2006 to February 2007. 
The following exclusion criteria were applied: non-Brazilian, inability or refusal to give informed consent, or immunodeficiency (including AIDS/HIV infection checked in medical records)

The study protocol was submitted and approved by the research ethics committees of the HMLMB, the Ludwig Institute for Cancer Research in São Paulo and the National Ethical Committee - CONEP (number 188/2006).

Women were recruited to take part in the study during the postdelivery follow-up period in the hospital. Eligible women were contacted by a health professional (a nurse or physician) and asked whether they wanted to know about a study on prevention of cancer of the cervix; those interested received full information about the study procedures and had a postnatal visit scheduled within 43-60 days after delivery to be enrolled in the study.

\section{Data and specimen collection}

During the routine postnatal visit women were enrolled and interviewed in an appropriate setting ensuring privacy, using a structured epidemiological questionnaire that included information about demographic characteristics, sexual behaviour, reproductive history, contraceptive practice and smoking habits. After the interview, a routine pelvic examination was conducted during the same visit by a gynaecologist/obstetrician and a cervical sample taken using a liquid-based cytology system 3 (DNA Citoliq, OIAGEN, Brazil) used to prepare a slide for Pap testing and for HPV DNA analysis.

\section{Liquid-based cytology}

Cervical samples were collected using the brush provided by the liquid-based DNA-Citoliq System (OIAGEN), immersed in UCM (Universal Collection Medium, OIAGEN), and processed following the manufacturer's instructions. ${ }^{14}$ The residual material of each sample, about $500 \mu$ l, was used for HPV DNA analysis.

Cytological slides were read blindly (ALF) in the Department of Pathology of the School of Medicine of the University of São Paulo. All abnormal smears and a 10\% random sample of normal smears were revised by the cytopathologist (VAFA) and a consensus diagnosis was established. Cytological results were reported according to the Bethesda System Terminology. ${ }^{15}$

\section{HPV DNA test}

Detection of HPV DNA extracted from exfoliated cells was performed using a standardised PCR protocol, amplifying a highly conserved 450 base pair segment in the L1 viral gene with generic primers PGMY09/11, and a coamplification with the internal $\beta$-globin control using the primer pair G73/G74 that amplifies a 268 base pair fragment as previously described. ${ }^{16}$ In all 301 cervical samples subjected to multiplex PCR this target of the $\beta$-globin gene was successfully amplified.

HPV typing was determined by an in-house dot-blot hybridisation protocol capable of detecting 28 different HPV types and 4 HPV Linear Array Test (Roche Molecular Diagnostics, California, USA). Considered as high risk were HPV types 16, 18, 26, $31,33,35,39,45,51,52,53,56,58,59,66,67,68,70,73$, and 82; and low risk types $6,11,40,42,54,55,61,71,72,81,83,84$, CP6108. ${ }^{3}$ Appropriate precautions were taken to reduce the possibility of specimen contamination.

\section{Statistical analysis}

Detection rates of HPV infection using PCR were described as percentages with $95 \%$ CI. To estimate the association of HPV infection with selected risk factors, prevalence ratios (PR) and 95\% CI were calculated, with HPV infection as the dependent variable and various exposure factors as independent variables. Most independent variables were grouped into two or more categories. For ordered (categorical) exposure variables, tests for linear trend $\left(\chi^{2}\right.$ trend) in the PR were conducted by categorising the exposure variables and entering the continuous scores. Variables selected in univariate analysis at a 0.20 significance level were included in the multivariate analysis. PR and their 95\% CI were estimated using a Generalised Linear Model (GLM) with binomial distribution and log link function. ${ }^{17}$ Statistical significance was assessed using the likelihood ratio test. ${ }^{18} \mathrm{~A}$ twosided $p$ value of less than 0.05 was considered to indicate statistical significance. All analyses were performed using STATA version 8.2. ${ }^{19}$

\section{RESULTS}

A total of 509 women were invited to participate: 24 refused, 163 women previously interested in participating did not return to the postnatal visit and 322 attended the postnatal visit. However, 18 women were not eligible (11 attended the postnatal visit after 60 days from delivery, one had HIV positive serology, one had had more than one delivery and five were over 24 years old). Three eligible women were excluded by the investigators: one had an acute infectious disease and two, aged $<18$ years, lived in a reformatory and had difficulty obtaining a legal representative signature of informed consent form within the study period. Therefore, 301 primiparous women were included in the analysis.

Participants and non-respondents were compared using medical hospital records. No statistically significant differences were found according to age $(p=0.205)$, marital status $(p=0.480)$, smoking habits $(p=0.183)$, prenatal healthcare $(p=0.436)$ and number of prenatal healthcare visits $(p=0.214)$.

The mean age of study participants was 19.9 years (median 20.0 years). Over $60 \%$ were white and more than $80 \%$ were married or living with the partner. Most women (266, 88\%) had at least 8 years (First Grade) of schooling. Less than one-third reported income (including earnings of all family members) equivalent to four or more minimum wages per month (equivalent to US\$ 479.20 during the study period).

In 11 women the Pap cytology was unsatisfactory for interpretation. Of the 290 women with satisfactory smears, 12 (4.1\%) had abnormal results: atypical squamous cells of undetermined significance (ASCUS) were found in five women; lowgrade squamous intraepithelial lesion (LSIL) in six women; and high-grade squamous intraepithelial lesion (HSIL) in one woman. These women are being followed and treated, if necessary. The single HSIL cytological case was submitted to colposcopy and cervical biopsy that showed cervical intraepithelial neoplasia grade 3 (CIN3) with glandular extension; a cold knife cone of cervix was performed and histological evaluation confirmed CIN3.

HPV DNA was positive in 11 of 12 women with abnormal cytological results, in all LSIL cytological cases and in the single HSIL cytological case only high-risk HPV types were detected, respectively, HPV types 16, 31, 33, 51, 52, 58, 68 and HPV 58. Of the five women with ASCUS: two had high-risk HPV types (33, 51, 73), two had only low-risk HPV types (HPV 54, 61) and one was HPV DNA negative.

Overall, HPV DNA was detected in 58.5\% (95\% CI 52.7\% to $64.0 \%$ ) of the women included in the analysis. The most common HPV types were HPV 16 (12.0\%), HPV 51 (10.0\%), HPV 52 (7.0\%), HPV 58 (6.0\%) and HPV 71 (6.0\%). HPV 18, 
a high-risk type present in bivalent and quadrivalent HPV vaccines, was detected in 2.3\%, and HPV 6 and 11, the low-risk types present in quadrivalent HPV vaccine, were detected in $4.3 \%$ of study participants (table 1$)$. Fifty-two (17.3\%) women were positive for any of the HPV types 6, 11, 16 and 18, and 40 women $(13.3 \%)$ were positive for HPV 16 or 18 . No woman was positive for all the four types present in quadrivalent vaccine. High-risk types were found in 133 (44.2\%) women, whereas 43 women $(14.3 \%)$ had only low-risk HPV types. One-hundred and two women $(33.9 \%)$ were positive for a single HPV type, 43 women $(14.3 \%)$ had two types, 18 women $(6.0 \%)$ had three and 13 women $(4.3 \%)$ had four or five types detected.

Prevalence ratios for HPV infection according to sociodemographic characteristics and smoking habits are shown in table 2. HPV prevalence decreased with increasing age ( $p$ for trend $=0.015$ ). Women with 11 or more years of schooling had lower prevalence

Table 1 Prevalence of human papillomavirus (HPV) DNA types among 301 young primiparous women, São Paulo, Brazil, 2006-07

\begin{tabular}{|c|c|c|}
\hline HPV DNA & $\mathbf{N}$ & $\%$ \\
\hline Negative & 125 & 41.5 \\
\hline Positive & 176 & 58.5 \\
\hline Single infection & 102 & 33.9 \\
\hline Multiple infections & 74 & 24.6 \\
\hline \multicolumn{3}{|l|}{ High-risk type } \\
\hline 16 & 36 & 12.0 \\
\hline 18 & 7 & 2.3 \\
\hline 26 & 7 & 2.3 \\
\hline 31 & 12 & 4.0 \\
\hline 33 & 6 & 2.0 \\
\hline 35 & 6 & 2.0 \\
\hline 39 & 2 & 0.7 \\
\hline 45 & 7 & 2.3 \\
\hline 51 & 30 & 10.0 \\
\hline 52 & 21 & 7.0 \\
\hline 53 & 16 & 5.3 \\
\hline 56 & 3 & 1.0 \\
\hline 58 & 18 & 6.0 \\
\hline 59 & 12 & 4.0 \\
\hline 66 & 6 & 2.0 \\
\hline 67 & 2 & 0.7 \\
\hline 68 & 8 & 2.7 \\
\hline 70 & 2 & 0.7 \\
\hline 73 & 8 & 2.7 \\
\hline 82 & 3 & 1.0 \\
\hline Any & 133 & 44.2 \\
\hline \multicolumn{3}{|l|}{ Low-risk type } \\
\hline $6 / 11$ & 13 & 4.3 \\
\hline 40 & 1 & 0.3 \\
\hline 42 & 3 & 1.0 \\
\hline 54 & 8 & 2.7 \\
\hline 55 & 5 & 1.7 \\
\hline 61 & 5 & 1.7 \\
\hline 71 & 18 & 6.0 \\
\hline 72 & 1 & 0.3 \\
\hline 81 & 2 & 0.7 \\
\hline 83 & 6 & 2.0 \\
\hline 84 & 9 & 3.0 \\
\hline CP6108 & 3 & 1.0 \\
\hline Only low-risk & 43 & 14.3 \\
\hline \multicolumn{3}{|c|}{ Vaccine-related HPV types } \\
\hline 6 and 11 or 16 or 18 & 52 & 17.3 \\
\hline 16 or 18 & 40 & 13.3 \\
\hline $16+18$ & 3 & 1.0 \\
\hline
\end{tabular}

(50\%) than those with less years of schooling (65\%). The HPV prevalences in former and current smokers were $60.0 \%$ and $88.0 \%$ respectively. Having ever smoked was significantly associated with HPV infection (PR 1.54, 95\% CI 1.30 to 1.83 ).

Regarding sexual behaviour, reproductive characteristics and contraception history, HPV prevalence increased with increasing number of lifetime sexual partners ( $p$ for trend=0.005) (table 3). The prevalence ratio of HPV infection for women who had had first sexual intercourse at 15 years of age or less compared to women over 15 years old was 1.14 (0.95 to 1.38). Previous history of sexually transmitted disease, previous miscarriage/ abortion, ever use of contraception, condom or hormonal contraception were not associated to HPV infection (table 3).

All selected variables (age, years of schooling, smoking habits, age at first sexual intercourse and number of lifetime sexual partners) were included in the multivariate analysis model. However, when age and years of schooling were included, the model did not converge. Following this, the variable 'years of schooling' was taken out. In the analysis including the other four variables, age at first sexual intercourse and number of lifetime sexual partners were not significantly associated with HPV infection, ( $p=0.117$ and $p=0.058$ respectively). The multivariate analysis revealed that only age ( $p$ for trend $=0.020$ ) and smoking habits $(p<0.001)$ were independently associated with HPV infection. Prevalence ratio of HPV infection for women aged $19-21$ years was 0.87 (95\% CI 0.72 to 1.05$)$ and for women $22-24$ years old was 0.78 (95\% CI 0.62 to 0.97 ) (reference group: women 15-18 years old). Women who reported ever having smoked were more likely to be infected (PR 1.50, 95\% CI 1.27 to 1.78 ) compared to women who never smoked.

Table 2 Prevalence ratios and corresponding 95\% Cl for human papillomavirus (HPV) infection according to selected sociodemographic characteristics and smoking among 301 young primiparous women, São Paulo, Brazil, 2006-07

\begin{tabular}{|c|c|c|c|c|c|}
\hline & Total & HPV+ & Prevalence ratio & $95 \% \mathrm{CI}$ & $\mathbf{p}$ \\
\hline Age (years)§ & & & & & 0.015 \\
\hline $15-18$ & 90 & $68.9 \%$ & 1 & & \\
\hline $19-21$ & 119 & $56.3 \%$ & 0.82 & 0.66 to 1.01 & \\
\hline $22-24^{*}$ & 92 & $51.1 \%$ & 0.74 & 0.58 to 0.95 & \\
\hline Ethnic group & & & & & 0.714 \\
\hline White & 190 & $57.9 \%$ & 1 & & \\
\hline Black & 31 & $54.8 \%$ & 0.95 & 0.67 to 1.33 & \\
\hline Mulatto & 77 & $62.3 \%$ & 1.07 & 0.87 to 1.33 & \\
\hline Indian & 3 & $33.3 \%$ & 0.58 & 0.12 to 2.87 & \\
\hline Years of schooling & & & & & 0.006 \\
\hline$<11$ & 168 & $65.5 \%$ & 1 & & \\
\hline$\geq 11$ & 133 & $49.6 \%$ & 0.76 & 0.62 to 0.93 & \\
\hline Marital status & & & & & 0.201 \\
\hline Living with partner & 245 & $56.7 \%$ & 1 & & \\
\hline Single & 56 & $66.1 \%$ & 1.16 & 0.94 to 1.45 & \\
\hline Income†,§ & & & & & 0.221 \\
\hline$<1$ & 13 & $69.2 \%$ & 1 & & \\
\hline $1-3$ & 188 & $59.6 \%$ & 0.86 & 0.59 to 1.26 & \\
\hline $4-6$ & 80 & $58.8 \%$ & 0.85 & 0.57 to 1.27 & \\
\hline $7-10$ & 11 & $45.5 \%$ & 0.66 & 0.31 to 1.38 & \\
\hline$>10$ & 5 & $40.0 \%$ & 0.58 & 0.19 to 1.79 & \\
\hline Smoking habits & & & & & 0.001 \\
\hline Never & 231 & $51.9 \%$ & 1 & & \\
\hline Ever‡ & 70 & $80.0 \%$ & 1.54 & 1.30 to 1.83 & \\
\hline
\end{tabular}

321 
Table 3 Prevalence ratios and corresponding 95\% $\mathrm{Cl}$ for human papillomavirus (HPV) infection according to selected sexual behaviour, reproductive characteristics and history of contraception among 301 young primiparous women, São Paulo, Brazil, 2006-07

\begin{tabular}{|c|c|c|c|c|c|}
\hline & Total & $\begin{array}{l}\mathrm{HPV} \\
+ \\
+\end{array}$ & $\begin{array}{l}\text { Prevalence } \\
\text { Ratio }\end{array}$ & $95 \% \mathrm{Cl}$ & $\mathbf{p}$ \\
\hline Age at first sexual intercourse (years) & & & & & 0.170 \\
\hline$>15$ & 169 & $55.0 \%$ & 1 & & \\
\hline$\leq 15$ & 132 & $62.9 \%$ & 1.14 & 0.95 to 1.38 & \\
\hline No of lifetime sexual partners $\dagger$ & & & & & 0.005 \\
\hline 1 & 120 & $50.8 \%$ & 1 & & \\
\hline $2-3$ & 116 & $58.6 \%$ & 1.15 & 0.91 to 1.46 & \\
\hline$\geq 4$ & 65 & $72.3 \%$ & 1.42 & 1.13 to 1.79 & \\
\hline Previous STD & & & & & 1.000 \\
\hline No & 292 & $58.6 \%$ & 1 & & \\
\hline Yes & 9 & $55.6 \%$ & 0.95 & 0.52 to 1.71 & \\
\hline Previous miscarriage/abortion & & & & & 0.668 \\
\hline 0 & 282 & $58.2 \%$ & 1 & & \\
\hline $1-2$ & 19 & $63.2 \%$ & 1.09 & 0.76 to 1.55 & \\
\hline Contraception & & & & & 0.638 \\
\hline Yes & 227 & $57.7 \%$ & 1 & & \\
\hline No & 74 & $60.8 \%$ & 1.05 & 0.85 to 1.31 & \\
\hline Condom use* & & & & & 0.246 \\
\hline Yes & 127 & $54.3 \%$ & 1 & & \\
\hline No & 100 & $62.0 \%$ & 1.14 & 0.91 to 1.42 & \\
\hline Hormonal contraception* & & & & & 0.654 \\
\hline Yes & 148 & $58.8 \%$ & 1 & & \\
\hline No & 79 & $55.7 \%$ & 0.95 & 0.75 to 1.20 & \\
\hline
\end{tabular}

STD, sexually transmitted disease.

*Only women who reported ever use of contraception. $+\chi^{2}$ for trend.

The prevalence ratio for the association between smoking and HPV prevalence had little variation after adjustment for socioeconomic or sexual activity status.

\section{DISCUSSION}

The HPV prevalence (58.5\%) found in the young primiparous women included in the present study was higher than those reported in women $<25$ years old, in previous population-based surveys, varying from $7.0 \%$ to $33.8 \% .^{20-24}$ Indeed, the present data indicate an HPV prevalence twofold higher than that reported in women younger than 25 years $(27.9 \%)$, in Brazil. ${ }^{25}$ In addition, two Brazilian cohort studies also revealed lower HPV prevalence at enrolment - $13.8 \%$ (mean age 33.3 years) and $24.6 \%$ (mean age 43 years). ${ }^{26}{ }^{27}$ However, these cohort studies did not focus on primiparous women, and, notably, the mean age of participants was substantially higher than in the present study.

Nevertheless, similar high HPV prevalence rates of $68.0 \%$ and $73.2 \%$ were reported, respectively, in sexually experienced young women aged 13-26, in Cincinnati, USA, and in young women self-referring for gynaecological care in Kampala, Uganda (12-24 years old, HIV-negative). ${ }^{28} 29$ Interestingly, Banura and colleagues in Uganda included 150 pregnant women, and found that concurrent pregnancy was significantly associated with higher prevalence of HPV infection (82.8\%), as compared to nonpregnant women. ${ }^{29}$ Recently, the same authors reported a similar HPV prevalence of about $60 \%$, as found in the present study, in early pregnancy, late pregnancy and after delivery in African first-pregnant young women, less than 25 years old (7.3\% HIV-positive). ${ }^{30}$

Although the present study did not have a concurrent control group for HPV prevalence in non-pregnant women, a study carried out previously in the same hospital, reported a lower prevalence $(27.1 \%)$ of high-risk HPV types in non-pregnant women $<25$ years, than in women of the present analysis. ${ }^{31}$

Thus, the high HPV prevalence in primiparous women in the present analysis is consistent with other reports and with the hypothesis that the physiological processes established during pregnancy modify the host-immune response and hormone status resulting in an increased prevalence of HPV infection measured in neonatal periods. Indeed, pregnant women are considered to be in an 'immunodepressed state' to facilitate survival of the 'semi-allogeneic' fetus. ${ }^{13} 32$

The analysis of type-specific HPV prevalence in young primiparous women shows that the proportion of participants infected with at least one high-risk HPV type (44.2\%) was higher than the proportion infected exclusively by low-risk HPV types $(14.3 \%)$, in agreement with previous reports. ${ }^{22-24}$ Furthermore, the present findings also agree with the prevalence of $49.5 \%$ for high-risk HPV types in pregnant women younger than 25 years, reported in Mexico. ${ }^{32}$ In the present study, among the participants positive for HPV, $42.0 \%$ had multiple HPV-type infection, in accordance with rates found in infected women in previous studies. ${ }^{24} 2829$

The HPV 16 prevalence of $12.0 \%$ found in the present study, is comparable to the findings from previous surveys in Latin America, varying from $8.2 \%$ to $13.7 \%,{ }^{5} 2122$ as well as the prevalence of other common HPV types - 51, 52 and 58 reported in Latin American women. ${ }^{21} 22$ In Brazil, HPV 16 was also the predominant type detected at baseline in two different cohort studies $(3.4 \%$ and $18.6 \%) .{ }^{26} 27$

The HPV 18 prevalence of $2.3 \%$ and the combined HPV 6 and 11 prevalence of $4.3 \%$, found in the present analysis, is similar to those reported in other Latin American studies in young females (16-24 years old), being 3.0\% and $4.4 \%$ respectively. ${ }^{5}$ Only $1.0 \%$ of participants in the present study had evidence of concomitant infections by high-risk HPV types 16 and 18, and none had simultaneously all the four HPV types included in one of the HPV vaccines, indicating that this group of young women could benefit from HPV vaccination.

Interestingly, only $17.3 \%$ and $13.3 \%$ of the participants were positive, respectively, for at least one of the HPV types included in the quadrivalent vaccine and for the two oncogenic HPV types, HPV 16 and 18, included in both vaccines suggesting that the majority of this group could still draw full benefit from catch-up HPV vaccination and protection against cervical cancer.

However, it is very important to consider that as most of study participants had been sexually active for years, a proportion of them may have been infected and cleared previous HPV infections. It is possible that although only $17 \%$ of the sample had HPV DNA detectable to any of the vaccine-specific types, some of the remaining $83 \%$ could have been exposed previously, cleared their infection, developed natural immunity and no longer had HPV DNA detectable at the cervix.

Furthermore, it is very important to stress that from a public health perspective, routine HPV vaccination should ideally be administered before sexual debut or shortly thereafter to achieve optimal vaccine effectiveness. The Advisory Committee on Immunization Practices, of the Centers for Disease Control in the United States, recommend routine HPV vaccination of girls at age 9-12 years and catch-up vaccination for females aged 13-26 years who have not been previously vaccinated or who have not completed the full immunisation series. ${ }^{7}$ In Brazil, HPV vaccination has been approved by regulatory authorities for females aged $9-26$ years but it is not yet included in public vaccination programmes. ${ }^{33}$ 
The postpartum period is a time when many routine interventions are provided to mothers, therefore, first delivery healthcare services could represent a suitable opportunity to offer HPV vaccines for adolescents and young women who have not been previously vaccinated using infrastructures already in place in health services, such as postdelivery follow-up period at maternity, postpartum visits and family planning services.

The relationship between HPV positivity and several risk factors for cervical cancer was also studied. The multivariate analysis highlights two risk factors: being a smoker (former or current) remains a risk factor, and increasing age was found to be inversely associated with HPV prevalence. Accordingly, two recent studies reported that current smoking was associated with an increased prevalence of HPV infection. ${ }^{34}{ }^{35}$ In fact, smoking has been reported to impair immune responses, which could lead to local immunosuppression facilitating the establishment or persistence of HPV infections. ${ }^{36}$ However, this association was reported in some studies but not corroborated by other studies. ${ }^{21} 2937$ In order to minimise possible bias, current smoker and former smoker were considered as just one category in this study. Because pregnant smokers are under pressure from health professionals and society to stop this habit, some women could have a tendency to classify themselves as former smoker.

The observed trend of decreasing HPV DNA prevalence with increasing age is in line with several studies that showed highest infection rates in younger women, with a linear decline of HPV infection risk with increasing age. ${ }^{12} 1337$

The main limitation of this study is that $32 \%$ of eligible women, previously interested in participating, did not return to the postnatal visit. The likely reason for the relatively large number of women not attending the postnatal visit at the hospital under study is the availability of postnatal services in public healthcare centres near the women's homes, therefore women may have preferred to attend those for practical reasons. As information about important factors such as age at first sexual activity and number of sexual partners were not available from delivery medical records, participants and all non-participants could not be compared according these variables. However, comparing participants and 32 (17\%) non-participant women that returned to the hospital for other medical appointments after the end of the study, no statistically significant difference was found in number of sexual partners $(p=0.085)$. In addition, participating and non-participating women did not differ according to other several factors. Therefore, the reported results would not be likely to have been significantly changed.

\section{CONCLUSIONS}

The present findings show that young primiparous women seeking public healthcare services had high cervical HPV prevalence, highlighting that these women are a high-risk group for cervical cancer development. As these young women also may represent a population with high school dropout rates, and hence are unlikely to benefit from potential school vaccination programmes, exploring alternatives to provide HPV vaccination to women at risk is warranted. Despite the relatively elevated prevalence of high-risk HPV infections detected in young primiparous women after delivery, only $13 \%$ of participants were positive for the oncogenic high-risk types included in vaccines - HPV 16 or 18 - indicating that the majority of these women would benefit from protection conferred by catch-up HPV vaccination programmes if provided at postnatal visits.

\section{What is already known on this subject}

- Human papillomavirus (HPV) vaccination should take place before potential exposure to HPV through sexual contact to achieve optimal vaccine effectiveness.

- However, vaccine coverage in adolescents can be low because they generally seek recommended preventive health services less frequently than other age groups.

- Therefore, opportunities for catch-up vaccination are valuable to improve coverage among the at-risk young female population.

\section{What this study adds}

Young primiparous women seeking public healthcare services had high cervical human papillomavirus (HPV) prevalence, highlighting that these women are a high-risk group for cervical cancer development.

- However, the prevalence of oncogenic high-risk types included in HPV vaccines was relatively low indicating that this group could benefit from catch-up HPV vaccination in the postnatal period.

Hence, this study supports that this population would derive benefit from catch-up vaccination and represents a target population for effective primary and secondary cervical cancer prevention programmes.

Acknowledgements The authors are grateful to Dr M.T. Aguado for helpful discussions. We are also grateful to Dr Corintio Mariani Neto for the unconditional support provided during field work and to nurses from Hospital Maternidade Leonor Mendes de Barros. The authors thank João Sobrinho Neto and José Carlos Prado from the Ludwig Institute for Cancer Research in São Paulo for HPV DNA testing by dot-blot hybridisation and Dr Gerson Bottacini das Dores from the Digene do Brazil (presently OIAGEN) for the DNA Citoliq system.

Funding This study was supported by a research grant from Department of Immunisation, Vaccines and Biologicals, World Health Organization (ref: V20-181-13).

Competing interests Luisa Lina Villa is a consultant and speaker for the Quadrivalent HPV Vaccine of Merck Sharp \& Dohme. José Eluf-Neto has served as a consultant to GlaxoSmithKline in 2006. The others authors have no potential conflicts of interest to disclose.

Ethics approval The study protocol was submitted and approved by the research ethics committees of the Hospital Maternidade Leonor Mendes de Barros, the Ludwig Institute for Cancer Research in Sao Paulo and the National Ethical Committee CONEP (number 188/2006).

Provenance and peer review Not commissioned; externally peer reviewed.

\section{REFERENCES}

1. Walboomers JMM, Jacobs MV, Manos MM, et al. Human papillomavirus is a necessary cause of invasive cervical cancer worldwide. J Pathol 1999;189:12-19.

2. Instituto Nacional de Câncer (INCA). Coordenação de Prevenção e Vigilância. Estimativa 2008. Rio de Janeiro: Incidência de Câncer no Brasil, 2008. http://www. 5 inca.gov.br/estimativa/2008/versaofinal.pdf.

3. de Villiers EM, Fauquet C, Broker TR, et al. Classification of papillomaviruses. Virology 2004:324:17-27.

4. Muñoz N, Bosch FX, De Sanjose S, et al. Epidemiologic classification of human papillomavirus types associated with cervical cancer. N Engl J Med 2003:348:518-27.

5. Perez G, Lazcano-Ponce E, Hernandez-Avila M, et al. Safety, immunogenicity, and efficacy of quadrivalent human papillomavirus (types $6,11,16,18$ ) L1 virus-likeparticle vaccine in Latin American women. Int J Cancer 2008;122:1311-18.

6. Paavonen J, Jenkins D, Bosch FX, et al. HPV PATRICIA study group. Efficacy of a prophylactic adjuvanted bivalent $\mathrm{L} 1$ virus-like-particle vaccine against infection with 
human papillomavirus types 16 and 18 in young women: an interim analysis of a phase III double-blind randomised controlled trial. Lancet 2007;369:2161-70.

7. Markowitz LE, Dunne EF, Saraiya M, et al. Centers for Disease Control and Prevention. Quadrivalent human papillomavirus vaccine: recommendations of the Advisory Committee on Immunization Practices (ACIP). MMWR Recomm Rep 2007:56:1-24.

8. Hildesheim A, Herrero R, Wacholder $S$, et al. Effect of human papillomavirus 16/18 $\mathrm{L} 1$ viruslike particle vaccine among young women with preexisting infection: a randomized trial. JAMA 2007;298:743-53.

9. Opel DJ, Diekema DS, Marcuse EK. A critique of criteria for evaluating vaccines for inclusion in mandatory school immunization programs. Pediatrics 2008;122:504-10.

10. Drain PK, Holmes KK, Hughes JP, et al. Determinants of cervical cancer rates in developing countries. Int J Cancer 2002;100:199-205.

11. Chan PK, Chang AR, Tam WH, et al. Prevalence and genotype distribution of cervical human papillomavirus infection: comparison between pregnant women and nonpregnant controls. J Med Virol 2002;67:583-8.

12. Smith EM, Ritchie JM, Yankowitz J, et al. HPV prevalence and concordance in the cervix and oral cavity of pregnant women. Infect Dis Obstet Gynecol 2004;12:45-56.

13. Takakuwa K, Mitsui T, Iwashita M, et al. Studies on the prevalence of human papillomavirus in pregnant women in Japan. J Perinat Med 2006;34:77-9.

14. Syrjanen K, Naud P, Derchain S, et al. Comparing Pap smear cytology, aided visual inspection, screening colposcopy, cervicography and HPV testing as optional screening tools in Latin America. Study design and baseline data of the LAMS study. Anticancer Res 2005;25:3469-80.

15. Solomon D, Davey D, Kurman R, et al. Forum Group. The 2001 Bethesda System: terminology for reporting results of cervical cytology. JAMA 2002;287:2114-19.

16. Gravitt PE, Peyton CL, Alessi TQ, et al. Improved amplification of genital human papillomaviruses. J Clin Microbiol 2000:38:357-61.

17. McCullagh P, Nelder JA. Generalized linear models. 2nd edn. New York: Chapman and Hall, 1989.

18. Breslow NE, Day NE. Statistical methods in cancer research. Vol I. The analysis of casecontrol studies. Publ. no. 32. Lyon: International Agency for Research on Cancer, 1980

19. StataCorp. Stata statistical software: Release 8.2. College Station, TX: Stata Corporation, 2003.

20. Sanjose S, Almirall R, Lloveras B, et al. Cervical human papillomavirus infection in the female population in Barcelona, Spain. Sex Transm Dis 2003;30:788-93.

21. Herrero $\mathbf{R}$, Castle PE, Schiffman $M$, et al. Epidemiologic profile of type-specific human papillomavirus infection and cervical neoplasia in Guanacaste, Costa Rica. $J$ Infect Dis 2005;191:1796-807.

22. Lazcano-Ponce $\mathbf{E}$, Herrero R, Munoz N, et al. Epidemiology of HPV infection among Mexican women with normal cervical cytology. Int J Cancer 2001;91:412-20.

23. Thomas Jo, Herrero R, Omigbodun AA, et al. Prevalence of papillomavirus infection in women in Ibadan, Nigeria: a population-based study. Br J Cancer 2004;90:638-45.
24. Dunne EF, Unger ER, Sternberg M, et al. Prevalence of HPV infection among females in the United States. JAMA 2007;297:813-19.

25. Nonnenmacher B, Breitenbach V, Villa LL, et al. Genital human papillomavirus infection identification by molecular biology among asymptomatic women. Rev Saude Publica 2002;36:95-100

26. Franco EL, Villa LL, Sobrinho JP, et al. Epidemiology of acquisition and clearance of cervical human papillomavirus infection in women from a high-risk area for cervical cancer. J Infect Dis 1999:180:1415-23.

27. Rosa MI, Fachel JMG, Rosa DD, et al. Persistence and clearance of human papillomavirus infection: a prospective cohort study. Am J Obstet Gynecol 2008;199:617.e1-7.

28. Kahn JA, Rosenthal SL, Jin Y, et al. Rates of human papillomavirus vaccination, attitudes about vaccination and human papillomavirus prevalence in young women. Obstet Gynecol 2008;111:1103-10.

29. Banura C, Franceschi S, Doorn LJ, et al. Infection with human papillomavirus and HIV among young women in Kampala, Uganda. J Infect Dis 2008;197:555-62.

30. Banura C, Franceschi S, van Doorn LJ, et al. Prevalence, incidence and clearance of human papillomavirus infection among young primiparous pregnant women in Kampala, Uganda. Int J Cancer 2008;123:2180-7.

31. Rama CH, Roteli-Martins CM, Derchain SF, et al. Prevalence of genital HPV infection among women screened for cervical cancer. Rev Saude Publica 2008;42:123-30.

32. Hernández-Girón C, Smith JS, Lorincz A, et al. High-risk human papillomavirus detection and related risk factors among pregnant and nonpregnant women in Mexico. Sex Transm Dis 2005;32:613-18.

33. Agência Nacional de Vigilância Sanitária - ANVISA. Resolução $n^{\circ} 2.777$, de 24 de agosto de 2006. Vacina quadrivalente recombinante contra papilomavírus humanos. Ministério da Saúde 2006a. http://ftp://ftp.saude.sp.gov.br/ftpsessp/ bibliote/informe_eletronico/2006/iels.dezembro.06/iels236/U_PT-MS-GM3124 071206. pdf (accessed 21 December 2008).

34. Shin HR, Franceschi S, Vaccarella S, et al. Prevalence and determinants of genita infection with papillomavirus, in female and male university students in Busan, South Korea. J Infect Dis 2004;190:468-76.

35. Vaccarella S, Herrero R, Snijders PJ, et al. IARC HPV Prevalence Surveys (IHPS) Study Group. Smoking and human papillomavirus infection: pooled analysis of the International Agency for Research on Cancer HPV Prevalence Surveys. Int J Epidemiol 2008; $37: 536-46$.

36. Poppe WA, Peeters R, Drijkoningen $M$, et al. Cervical cotinine and macrophageLangerhans cell density in the normal human uterine cervix. Gynecol Obstet Invest 1996;41:253-9.

37. Giuliano AR, Papenfuss M, Abrahamsen M, et al. Human papillomavirus infection at the United States-Mexico border. Cancer Epidemiol Biomarkers Prev 2001;10:1129-36. 